\title{
Gap percolation in rainforests
}

\author{
Ricard V. Solé, Frederic Bartumeus and Javier G. P. Gamarra
}

Solé, R. V., Bartumeus, F. and Gamarra, J. G. P. 2005. Gap percolation in rainforests. - Oikos 110: 177-185.

\begin{abstract}
Rainforests biodiversity is sustained by the three-dimensional structure of their canopy which provides a wide range of physical microenvironments. Given the dynamic nature of the forest, the recognition of stable vertical layers or strata in the canopy is controversial. The spatial characterisation of potential habitats of understory species is not straightforward due to the complex structure of rainforest canopies and the wide ecological variability to which rainforest species can be adapted. Here we present a new description of potential understory habitats that give rise to a well-defined characteristic vertical scale of forest organization $\mathrm{h}_{\mathrm{c}} \approx 13 \mathrm{~m}$. Species living in microenvironments occurring at canopy heights below this critical height $h_{c}$ can only experience landscapes with disconnected habitat patches (i.e. fragmented habitat landscapes), while those species capable of living also above $h_{c}$ will experience a fully connected landscape of suitable microenvironmental conditions. The possible implications for plant dispersal and animal colonisation strategies living at the understory or close-to-floor are discussed in relation to rainforest gap-dynamics, habitat loss and habitat fragmentation processes. Longrange and directed dispersal strategies (e.g. plant seed dispersal by animals) are optimal for those species living below $h_{c}$, providing the best exploration of scarce habitats and a major robustness to habitat changes. On the other hand, dispersal strategies of those species capable to exploit habitats above $h_{c}$ need not to be based on directed long-range mechanisms. Different dispersal strategies may in turn imply different sensitiveness of species to habitat loss and habitat fragmentation processes in the rainforest.
\end{abstract}

R. V. Solé and F. Bartumeus, Complex Systems Lab-ICREA, Universitat Pompeu Fabra, Dr. Aiguader 80,ES-08003 Barcelona, Spain (ricard.sole@upf.edu). RVS also at: Santa Fe Institute, 1399 Hyde Park Road, NM 87501, USA. FB also at: Centre d'Estudis Avançats de Blanes CEAB, CSIC, Accés Cala Sant Francesc 14, ES-17300 Blanes, Girona, Spain. - J. G. P. Gamarra, Center for the Environment, Dept of Natural Resources, 103 Rice Hall, Cornell Univ. Ithaca, NY, USA.

Vertical organisation of trees and plants is a characteristic feature of rainforests. The so called canopy structure provides the forest with a three-dimensional structural matrix where a wide range of community functions must be accomplished. Thus, the comprehension of canopy structure characteristics actually provides an appropriate framework to study the relationship between structure and function in ecosystems (Richards 1952, Terborgh 1992, Whitmore 1997).

The canopy height variability of rainforests is a relevant descriptive factor of the changes in the quantity and quality of light determining microenvironment temperature, humidity and soil moisture (Denslow 1987, Becker et al. 1988). The structure of canopy heights has a very important role in shaping different microenvironments, that ultimately determine the threshold conditions that constraint plant growth and physiology (Zagt and Werger 1996, Leigh 1999), as well as physiological limits for many arboreal and terrestrial animals (Shelly 1984, Leigh 1999). These microenvironments strongly influence the competitive outcomes of different species living in the understory or the rainforest 
floor (Leigh and Wright 1990, Reagan 1992). Therefore, canopy height structure involves the three-dimensional organisation in space of a wide range of aboveground vegetation components (e.g. trees, shrubs, herbaceous plants, lichens, fungi) and animal life forms (e.g. amphibians, small reptiles and mammals, arthropods and invertebrates, Leigh 1999). Overall, canopy height structure should shape and constrain the biodiversity of understory and close-to-floor species (Gilbert 1980).

The traditional analysis of vertical forest architecture has been approached from the study of profile diagrams obtained from measurements of the trees in narrow strips of the forest (Richards 1952, Terborgh 1985). Since then, the vertical structure of forests is typically defined as layers or strata. The number of strata in rainforests is supposed to be greater than in temperate forests (Terborgh 1985). Moreover, conceptual models exist to explain the organisation of canopy forests in strata (Terborgh 1992). Nevertheless, strata are largely subjective and their exact demarcation is not obvious as the layers grade into each other. Recent techniques are making use of remote sensing analysis (such as laser altimetry) that allows to obtain well defined profiles based upon standardised measurements (Drake et al. 2002). The recognition, at different heights in the rainforest canopy, of different structures, species or environments to a degree that may define identifiable zones, is a useful aid for description or analysis but it has seldom received critical examination (Smith 1973, Baker and Wilson 2000, Parker and Brown 2000). Indeed, stratification can be considered a simplification and an abstraction given the dynamic nature of the forest. A forest is in a continuous state of flux, it consists of a mosaic of patches at all stages of the growth cycle (Whitmore 1997). The dynamical changes in time and space of canopy gaps modify the intermingling of tree crowns in their search for light, therefore screening a possible stratified organisation of plant species. In this context, the existence of universal and robust properties in the vertical structure of rainforests is still an unresolved question.

These complementary perceptions of the structure of forest canopy (as vertically stratified or as a mosaic of growing gaps) give rise to a different spatial characterisation of potential habitats for understory species. On the one hand, the 'static' vision is used to delimitate habitats in the vertical axis, emphasising the existence of specialist species adapted to live in certain canopy heights, and vertical competitive segregation processes of related species (Terborgh 1992, Reagan and Waide 1996). On the other hand, the 'dynamic' vision highlights the existence of strong relationships between understory species and certain growth cycle phases of the forest (i.e. gap-phase, building-phase and maturephase in Whitmore 1988). Thus, potential habitat differentiation is based on the XY-plane dimension of the forest. The typical light-demanding and shade tolerant species distinction comes from such a dynamical envision of the rainforest canopy (Welden et al. 1991, Chesson and Pantastico-Caldas 1994, Whitmore 1997, Schnitzer and Carson 2000, Molino and Sabatier 2001).

However, the survival of many species in the understory depends on a great complexity of intertwined factors that must be sorted within a broad ecological or environmental range of conditions. Thus, although the life-history traits of some rainforest species may be related to a very specific set of ecological conditions, a wider range of survival conditions is also possible for many species. In particular, the possibility of covering a given range of conditions by some species might influence their survival on several growth cycle phases or on different vertical strata. In these cases, we need more realistic habitat characterisations based on the integration of the whole range of microenvironmental conditions in which the species is able to survive.

To understand how canopy structure is related to habitat availability in species adapted to different ecological ranges (or microenvironmental conditions) we propose a new characterisation of rainforest understory habitats that integrates both previous envisions (i.e. static and dynamic) of rainforests. This characterisation of habitats takes into account the whole three-dimensional structure of vegetation by considering both the vertical layered structure on the $\mathrm{Z}$ axis, and the XY-plane mosaic structure itself. The habitat is defined as the whole set of microenvironments that exist below a maximum canopy height. The higher the maximum height, the wider the range of potential environmental conditions involved in the habitat. Thus, it is explicitly assumed: i) the capacity of many rainforest species to exploit different growth cycle phases as well as different canopy layers, and ii) the complex spatial pattern of understory microenvironments. Such a definition of habitat allows for the emergence of a well-defined critical height separating two domains in forest architecture. This critical height scale is obtained from the analysis of the spatial distribution of low canopy points (Welden et al. 1991, Condit 1998) and reveals a threshold in the vertical organisation of the forest structure which is clearly related to the gap-dynamical aspects of the forest. The geometrical properties related to gap connectivity imposed by this threshold might have non-trivial consequences for dispersal or colonisation strategies of animals and plants within the understory or close to the forest floor. By means of a Lévy flight model the effect of a percolation threshold on dispersal strategies is illustrated. 


\section{Barro Colorado Island plot}

We have used data on canopy height taken from the Barro Colorado Island (BCI) 50-ha plot (Hubble and Foster 1986, Condit 1998) that correspond to annual censuses of $5 \times 5 \mathrm{~m}$ resolution made from 1985 to 1993. Large forest plot research projects provide large sets of data collected using standardised techniques. The method used to estimate canopy height is specified in Hubble and Foster (1986) and Condit (1998). The canopy height data have already been used to map canopy gaps and their changes through time (Hubble and Foster 1986) and as a way to assess light environment for saplings below (Welden et al. 1991). We have defined low-canopy points (LCP) as those sites where the maximum canopy height is lower than some given threshold (h). Thus, a different set of LCP $(\Omega(h))$ will be obtained using different threshold heights. For each chosen height, we get a set of canopy sites and a set of low-canopy sites (i.e. LCP). Low-canopy sites may be named also "gap" sites although we must understand a "gap" in a broader sense than commonly used in rainforest studies. A set of gap sites may represent, in this case, a set of suitable patches conforming a three-dimensional habitat were a species can survive. As an example, for arboreal species, low-canopy maps may define a threshold height below which a range of suitable understory habitats or environmental conditions are possible, involving both different strata and growth cycle phases. Moreover, canopy height is a good surrogate of the behaviour of canopy light transmittance from the top to the bottom of the canopy in spite of the fact that other structural factors of the canopy such as the spatial distribution of trees and foliage could provide a potential source of variability of the understory light levels (Pelt and Franklin 2000). As a consequence, low-canopy maps (LCP spatial distribution) could also be shaping the landscape of potential available terrestrial microenvironments for some close-to-floor or terrestrial species (i.e. invertebrates and small vertebrates) strongly dependent on some light-related descriptors such as quantity and quality of light irradiance, temperature or humidity.

By progressively increasing the low-canopy cutoff by one-meter steps (from a minimum height of $1 \mathrm{~m}$ to a maximum of $35 \mathrm{~m}$ ), 35 different low-canopy maps can be obtained representing habitat availability landscapes for different species. In Fig. 1 examples of LCP maps are shown for three different cutoff heights (h). Black cells represent suitable (available) habitat. White cells represent non-suitable habitat. As expected, the number of suitable patches $N_{\omega}$ (h) grows with $h$. The set of LCP grows in size and appears to be correlated in space: the new LCP that appear as $\mathrm{h}$ grows tend to be close to the previous ones.
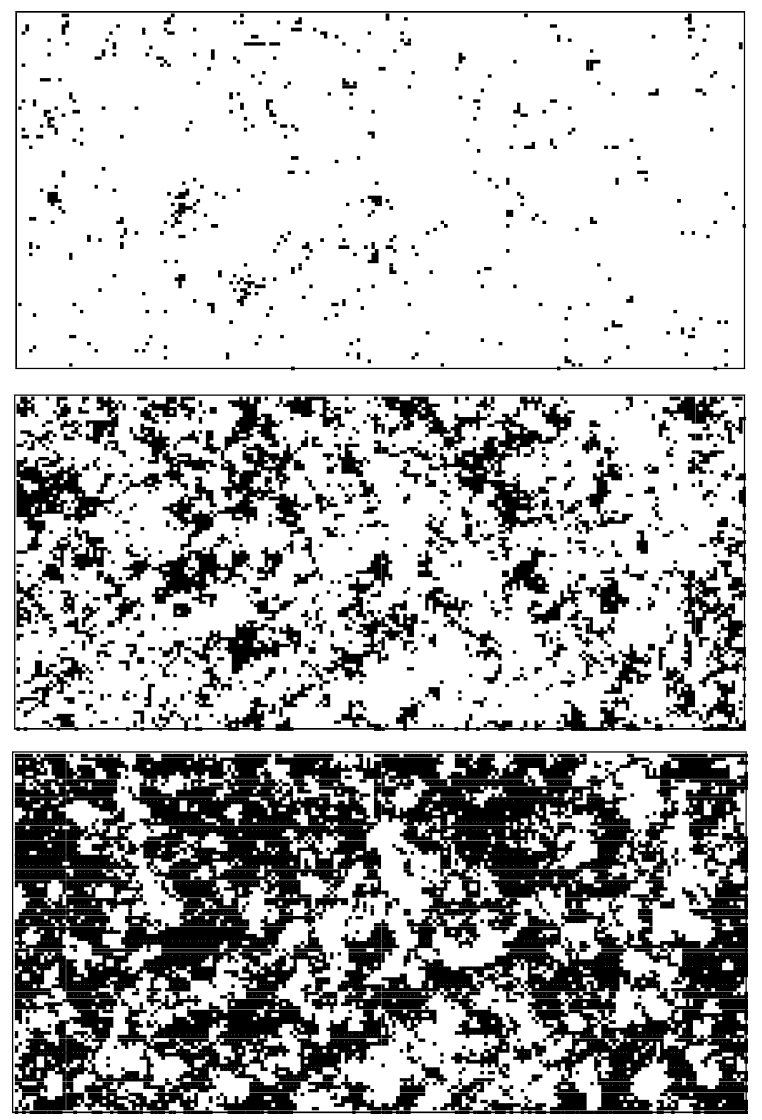

Fig. 1. Low-canopy maps from Barro Colorado 50-ha plot 1985 census data, see text. Black cells are low-canopy points (LCP) indicating suitable habitat. Non-suitable habitat is represented by white cells. LCP are $5 \times 5 \mathrm{~m}$ square sites such that their canopy is lower than some given threshold $\mathrm{h}$. Top: $\mathrm{h}=5 \mathrm{~m}$. Middle: $\mathrm{h}=10 \mathrm{~m}$. Bottom: $\mathrm{h}=15 \mathrm{~m}$. Close to $\mathrm{h}_{\mathrm{c}} \approx 13 \mathrm{~m}$ the set of LCP becomes connected.

\section{Percolation in low-canopy maps}

The analysis performed in this study involves the presence of a critical height at which canopy gaps percolate through the entire forest. Percolation is recognised as a key property in landscape ecology (Gardner et al. 1987, Turner et al. 2001). When sets of neighbouring, connected small-scale sites define a cluster spanning the entire system under consideration, such cluster is called a percolation cluster (Stauffer and Aharony 1985, Peitgen et al. 1992, Milne et al. 1996, Keitt et al. 1997). Percolation phenomena influence metapopulation dynamics (Andren 1994, Bascompte and Solé 1996, Wiens et al. 1997, With and King 1999), invasion processes (Loehle et al. 1996, With 2002), and are relevant to conservation biology (With 1997, Bunn et al. 2000) and ecosystem function (Gamarra and Solé 2002).

More precisely, let us consider the set of low-canopy points (LCP) $\Omega(\mathrm{h}$ ) that is defined for a given height $\mathrm{h}$. For each $\mathrm{h}$, a different set will be obtained. Fig. 2A 
shows the monotonic increase of $\mathrm{N}_{\omega}(\mathrm{h})$, defined as the number of points in the set $\Omega(\mathrm{h})$ as $\mathrm{h}$ grows. However, the geometrical properties displayed by $\Omega(\mathrm{h})$ are far from monotonous. One key property is the emergence of percolation at $\mathrm{N}_{\omega}\left(h_{c}\right)$ once a critical canopy height $h_{c}$ is reached. A very useful characterisation of the transition to percolation in a lattice is provided by the computation of the time required for a "fire" to propagate through the system (Stauffer and Aharony 1985). Let us assign an integer value $S(i, j)=1$ to each $L C P$ on $\Omega(h)$, and $\mathrm{S}(\mathrm{i}, \mathrm{j})=0$ otherwise. A simple burning algorithm is defined as follows: (1) at some time step $t=0$, the LCP at one edge of the low-canopy maps are burned, i.e. they take a new state $S(i, j)=2 ;(2)$ at the next step, those LCP that are nearest neighbours to burned sites are also burned. In this way, the fire propagates until no new LCP becomes burned (i.e. fire extinction). The total time required to complete the fire spreading $\mathrm{T}_{\mathrm{s}}$ (i.e. the time until fire extinction), is computed for different heights $h$. At percolation, $\mathrm{T}_{\mathrm{s}}(\mathrm{h})$ will present a maximum. For the BCI 50-ha plot the maximum $\mathrm{T}_{\mathrm{s}}(\mathrm{h})$ is reached at a height of $\mathrm{h} \approx 13 \mathrm{~m}$ (Fig. 2B). The presence of a maximum and the overall profile obtained in Fig. 2B is easily understood in terms of the complexity of the underlying landscape. For $h<h_{c}$ the fire hardly spreads due to the fragmented nature of $\Omega(\mathrm{h})$, therefore fire extinction is rapid and $T_{s}(h)$ is low. For $h>h_{c}$, a more or less continuous set of connected gap sites is available with a rather homogeneous structure, therefore the time until fire extinction is large. Just at the transition $h=h_{c}$, the landscape $\Omega(\mathrm{h})$ is a fractal object (Solé 2000) and the fire no longer propagates through a simple geometrical system. Instead, the percolation cluster displays complex features at multiple scales and the fire must follow such features over time. In a way, the transient time is tied to how dificult is to describe the object. The divergence in $\mathrm{T}_{\mathrm{s}}\left(\mathrm{h}_{\mathrm{c}}\right)$ is characteristic of complex systems at the phase transition points (Peitgen et al. 1992, Solé 2000).

In two-dimensional random uncorrelated landscapes, the spanning cluster of connected gaps would appear at a gap fraction $\mathrm{p}_{\mathrm{c}} \approx 0.40725$ (Stauffer and Aharony 1985). However, at the BCI 50-ha plot, the largest cluster shows off at a somewhat greater gap fraction (Fig. 2B). We simulated the fire spreading algorithm for both types of landscapes (i.e. the real BCI landscape and a random landscape with the same LCP). Above $h_{c}$, the landscape fractal dimension (box counting method) does not differ that of a purely random landscape $(\mathrm{D} \approx 2)$. Below it, gaps are closely correlated in space, so $\mathrm{T}_{\mathrm{s}}\left(\mathrm{h} ; \mathrm{h}<\mathrm{h}_{\mathrm{c}}\right)$ is higher than in random landscapes (Fig. 2B). The decrease in fractal dimension (Fig. 2C) can also be explained in terms of strongest correlations in gap
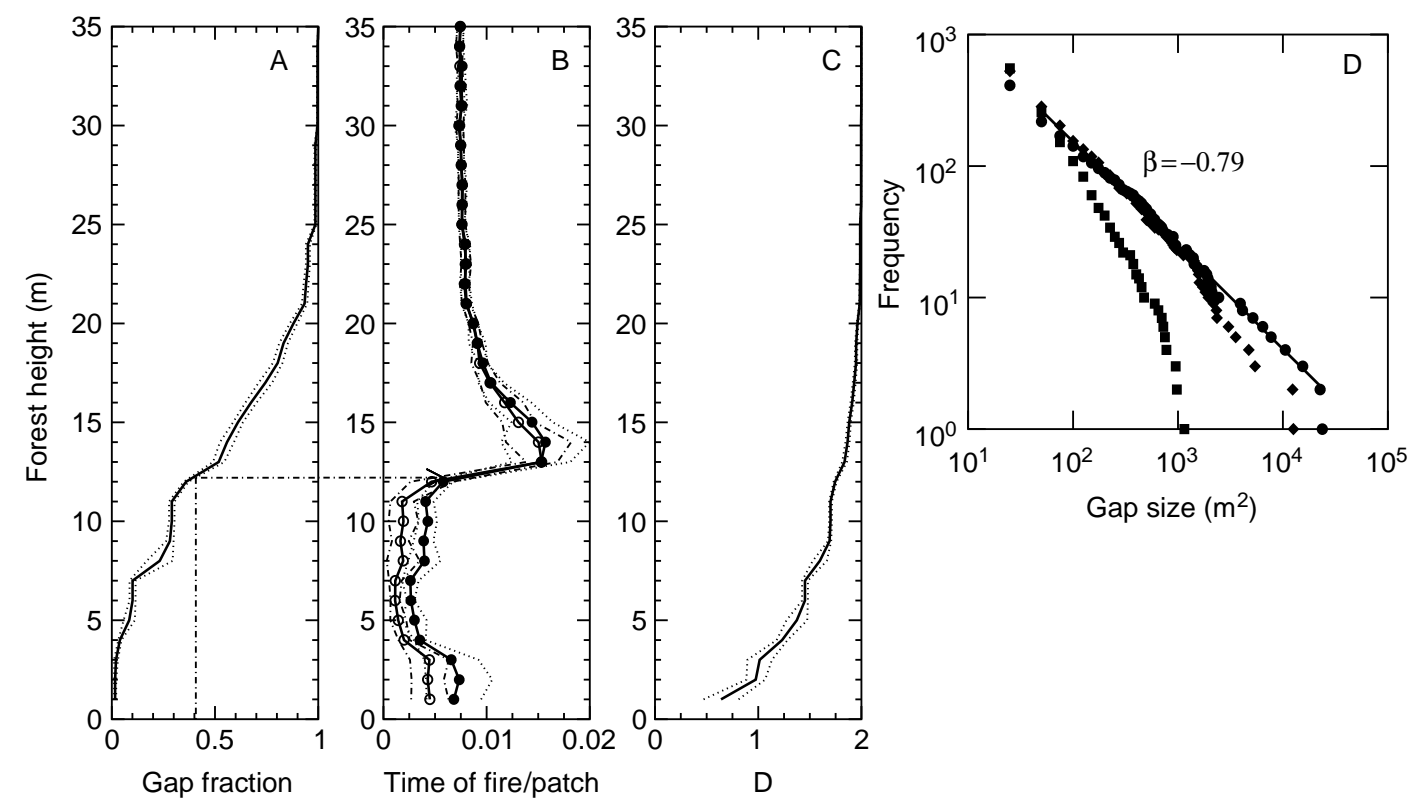

Fig. 2. Percolation in the BCI plot, as measured from the burning algorithm. A maximum is reached at $\mathrm{h} \approx 13 \mathrm{~m}$, indicating that a percolation cluster is present. (a) Gap fraction in relation to forest height. Gap fraction is defined as the fraction of LCP (or suitable sites) at every height (i.e. $\mathrm{N}_{\omega}(\mathrm{h}) / \mathrm{N}_{\text {total }}$ ). Dashed lines indicate confidence intervals $\alpha=0.95$ for a series of eleven years $1985-1995$. The arrow shows the height at which percolation should appear in a random landscape (lattice) under a Moore neighbourhood ( 8 nearest neighbours). In these type of lattices the critical value for the gap fraction is $p_{c} \approx 0.40725$. (b) Fire lifetime ( $T_{s}(h)$ ) in the burning algorithm. Black circles depict the results in the real landscape (1985 census data). White circles represent the results for 10 different random landscapes with the same number of LCPs. Dashed lines are confidence intervals for 10 different burning algorithms for every case. (c) Corresponding average fractal box-counting dimension plus confidence intervals for the whole 19851995 series. (d) Fractal behaviour close to the percolation threshold. The cumulative distribution of gap area sizes is shown for three different heights: $\mathrm{h}=8$ squares, $\mathrm{h}=10$ diamonds and $\mathrm{h}=14$ circles. As expected for a fractal, percolating cluster, a power law is obtained at $\mathrm{h} \approx \mathrm{h}_{\mathrm{c}}$, i.e. $\mathrm{N}_{>}(\mathrm{G}) \approx \mathrm{G}^{-\beta}$, with $\beta=0.79 \pm 0.02$ and thus, $\mathrm{N}(\mathrm{G}) \approx \mathrm{G}^{-\gamma}$ with $\gamma=\beta+1=1.79$. 
spatial distribution below $h_{c}$. These differences point to the greater importance of local connectivities close to the forest floor, due to larger spatial correlations. At $h_{c}$, a very large cluster of connected LCP emerges, allowing for long-range correlations through all the landscape. Moreover, the distribution of gap area sizes in the percolation threshold is fractal, that is, no characteristic area sizes are identifiable. In Fig. 2D the cumulative distribution $\mathrm{N}_{>}(\mathrm{G})$ is shown, defined as

$N_{>}(G)=\int_{G}^{\infty} N(G) d G$

where $N(G)$ is the number of gaps of size $G$. The shape of this distribution changes with $h$, and actually reveals a scaling law:

$\mathrm{N}(\mathrm{G}) \approx \mathrm{G}^{-\mathrm{y}} \exp \left(\mathrm{G} / \mathrm{G}^{*}\right)$

where $G^{*}$ is a given (h-dependent) cutoff. Once $h=h_{c}$ is reached, a shift from a set of LCPs with a characteristic length scale to a scale-free (fractal) pattern is observed. The presence of a power-law is consistent with the existence of a percolation phase transition.

\section{Dispersal strategies}

Given the previous results, and in relation to species habitat availability, we aim to know the metapopulation consequences of the percolating geometry observed in rainforest's architecture. The potential habitat availability for a species depends on two main factors: i) species biological constraints (i.e. physiological, behavioural or ecological) and ii) spatial and dynamical properties of the habitat. Within the biological constraints, both the species ecological ranges and their dispersal or colonisation strategies are of main importance in order to properly exploit the habitat (With and King 1999). Thus, a good question to be answered is, how does habitat loss and fragmentation influence habitat availability in species with different ecological ranges and different dispersal strategies?

A Lévy flight model has been introduced to study the effect of dispersal or colonisation processes in different availability landscapes (i.e. low-canopy maps) by rainforest plants or animals living in the understory (arboreal) or close-to-floor (terrestrial). Lévy flights are random walks characterised by the fact that the length of each successive step or jump $l_{j}$ varies according to a power law function of the form:

$\mathrm{P}\left(\mathrm{l}_{\mathrm{j}}\right)=\mathrm{l}_{\mathrm{j}}^{-\mu}$

with $1<\mu \leq 3$. Values $\mu \leq 1$ do not correspond to normalisable probability distributions. Thus, a Lévy flight has no intrinsic jump length scale, and jumps of seemingly very long length may be observed. The power law exponent $\mu$ describing the jump length distribution is named Lévy exponent or Lévy index defining a continuum of strategies from long-range to short-range dispersal. As the Lévy index increases from 1.1 to 3 extremely long jumps occur with less frequency, and thus a reduction in the overall dispersal scales is expected (from super-diffusive to Gaussian macroscopic diffusion). In particular, for $\mu \geq 3$ a typical Brownian dispersal strategy is recovered. Lévy flight patterns are common in large-scale animal movements (Viswanathan et al. 1999, Marell et al. 2002, Bartumeus et al. 2003), some of them inhabitants of the rainforests (RamosFernández et al. 2004).

For each of the low-canopy maps obtained, different dispersal or colonisation strategies were simulated by changing the Lévy exponent of Lévy flight distribution from 1.1 to 3 . Thus, the whole gradient from Lévy to Brownian strategies was covered. The results obtained are the same for both periodical and absorbent boundaries. The simulations proceed as follows: (1) an individual (or group of individuals) located in a suitable habitat is dispersed to another point of the landscape by randomly drawing a move or jump length from a Lévy flight distribution (i.e. truncated power-law with a range of jump lengths between 5 and $1000 \mathrm{~m}$ ); (2) if the cell represents a suitable habitat, the species occupy the new habitat. If it is a non-suitable habitat or if it is an habitat already occupied, the individual (or group) is believed to be non viable in that cell and dies. (3) From the pool of habitats occupied (both old an new habitats included) an individual (or group) is chosen at random and a new dispersal/colonisation event is executed. (4) The simulation finishes when a certain dispersal effort has been made by the whole dispersing population. This dispersal effort is measured in terms of a total dispersal distance traversed by the population.

In particular, we define the dispersal efficiency function $\eta(\mu)$ to be the inverse of the total distance traversed by the population (i.e. the inverse of dispersal effort), so that:

$\eta(\mu)=\frac{1}{<1>N}$

where, $\mathrm{N}$ is the mean number of flights taken in a Lévy dispersal process and $<1>$ is the mean dispersal-jump length of the process. Thus, dispersal efficiency is defined as in Viswanathan et al. (1999) but it is computed as a population dispersal efficiency and not as an individual searching efficiency. A low value of $\eta$ can result from either a large $\mathrm{N}$ or a large $<1>$, corresponding to large and small $\mu$, respectively. Small $\mu$ involves Lévy dispersal strategies based on few dispersal events but covering long dispersal distances, while large $\mu$ involves Brownian dispersal strategies that are based on a high number of local dispersal events. Thus, it is assumed that largerange dispersal involves higher time or energetic costs than short-range dispersal. Defining efficiency in such a 

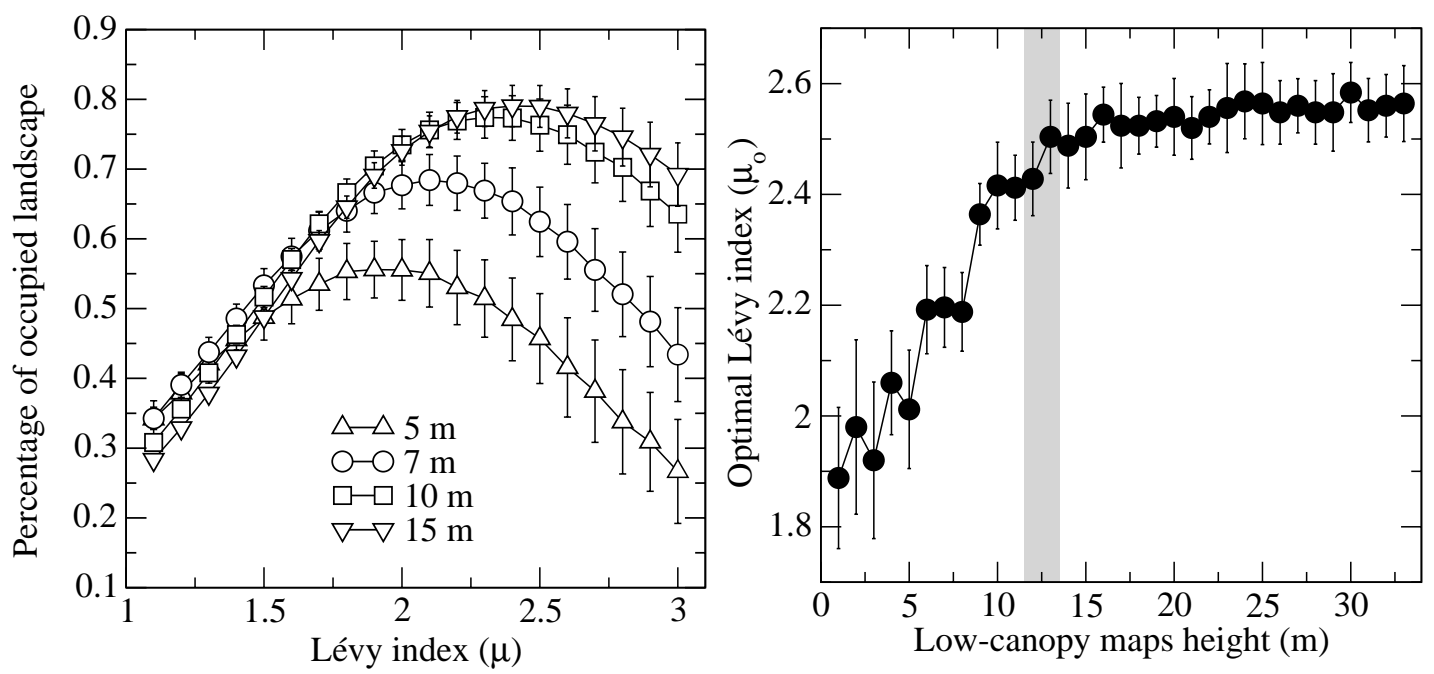

Fig. 3. Left panel: percentage of occupied landscape related to Lévy index exponent values ( $\mu$ ). Different low-canopy maps obtained at different heights (i.e. 5, 7, 10 and $15 \mathrm{~m}$ ) show different optimal Lévy indexes. When higher cutoff heights are chosen, and thus, larger suitable habitat is being considered, higher is the optimal Lévy index. Right panel: a change from low to high optimal Lévy indices $(\mu)$ representing a change from large-range to short-range dispersal strategies, is observed as the cutoff heights are increased from 1 to $35 \mathrm{~m}$. The asymptotic behaviour of this relationship is due to a percolation effect in the landscapes. Average and standard deviation values are computed from 25 curves obtained.

way, and given a certain amount of time (or distance travelled), dispersal strategies based on Lévy distributions with small Lévy indexes (i.e. $\mu \leq 1.5$ ) provide a reduced statistical sampling power while those based on large Lévy indexes (i.e. $\mu \geq 2.5$ ) provide a reduced spatial sampling power. For any landscape, the optimal Lévy index will always be within the intermediate range $1.5<\mu<2.5$. However, a unique optimal $\mu$ value emerges for a specific landscape configuration.

For a given dispersal efficiency we compute the percentage of new occupied landscape as the ratio of the number of new occupied landscape (i.e. number of suitable occupied cells previously non-occupied) to $\mathrm{N}_{\omega}$ (h) (i.e. number of suitable cells). In Fig. 3 (left), different habitat suitability landscapes show different optimal Lévy indexes $(\mu)$. As higher cutoff heights are chosen to obtain the low-canopy maps (i.e. from 5 to $15 \mathrm{~m}$ ), higher is the optimal Lévy index. Fig. 3 (right) shows the optimal Lévy indexes (i.e. those indexes showing the maximum percentage of suitable habitat occupied provided certain dispersal effort) for each of the 35 lowcanopy maps. As higher canopy cutoff heights are considered, more connected low-canopy maps are obtained, and a change from Lévy to Brownian dispersal strategies can be observed as we increase the cutoff height. An asymptotic behaviour related to the percolation effect is observed.

\section{Discussion}

Early studies of canopy structure suggested that the vertical organisation of canopy trees was layered and that some functional processes of rainforests should be dependent on this vertical layered structure (Richards 1952). However, some authors have recently shown some limitations of 'classical' stratification studies in relation to scale dependence, point of reference and spatial averaging. They suggest that it may be more fruitful to discard the presumption of stratification entirely (Parker and Brown 2000). However, it is the lack of objective quantitative measures that has prevented to rigorously define the presence of well-defined scales in rainforest spatial organisation.

In this study, we have shown that one of these possible scales can be described in terms of the percolation of canopy gaps once a critical canopy height $\left(\mathrm{h}_{\mathrm{c}}\right)$ is reached. Indeed, when forests height-class distributions are complex (i.e. highly continuous distributions) this percolating critical canopy height $\left(\mathrm{h}_{\mathrm{c}}\right)$ may provide useful structural and dynamical information about the forest. On the other hand, if height-class structure is simple (e.g. savannah forests) it may be better to identify the real strata (i.e. height classes) of the forest. Strictly speaking, the percolating critical canopy height $\left(\mathrm{h}_{\mathrm{c}}\right)$ is a topological measure that only depends on the height-class structure of the forests. We have to look at the physical and dynamical factors providing a given height-class distribution to understand the emergence of a specific $h_{c}$. The most important factors involved in height-class distributions are density, gap-dynamics, and external perturbances. In natural monospecific forests (i.e. single species forests with complex height-class distributions) this integrative measure may also provide comparable information on forest general status. 
Traditionally, theoretical analyses of gap-dynamics has been focused on the processes of gap formation and canopy recovery (Runkle 1984, Solé and Manrubia 1995, Kubo et al. 1996, Katori et al. 1998, Iwasa 2000). However, these studies are typically restricted to gaps defined at some arbitrarily chosen height. In an early study (involving the analysis of a single-height snapshot) the loss of spatial gap correlations at some given canopy height was explained in terms of tree fall dynamics (Solé and Manrubia 1995). More recently, the influence of neighboring sites on transition rates (i.e. from non-gap to gap sites and viceversa) has been studied in detail (Satake et al. 2004, Schlicht et al. 2004). It is known that the rate of transition from a non-gap to a gap site increases with the number of neighbours that are currently in the gap state (Hubble and Foster 1986, Kubo et al. 1996, Satake et al. 2004, Schlicht et al. 2004). This relationship may cause higher spatial correlations than expected from a pure random gap-formation process. However, the exact function relating the number of gap-state neighbours and the transition rates, as well as its possible dynamic consequences, have just started to be explored empirically and theoretically (Kubo et al. 1996, Satake et al. 2004, Schlicht et al. 2004).

Our results for the BCI rainforest, show that below $\mathrm{h}_{\mathrm{c}}$ there exist higher correlations than the ones given in a pure random percolation process (where LCP would show a random distribution). This higher correlations disappear at $\mathrm{h}>\mathrm{h}_{\mathrm{c}}$, consistently with a random-like distribution of crowns. Future models of gap-dynamics should take into account the presence of this critical height and the presence of non-random landscape correlations above the percolation height $\left(\mathrm{h}_{\mathrm{c}}\right)$, providing a dynamical explanation for both.

The network of relationships established by rainforest species to survive may involve several spectra of microenvironments or growth cycle phases. Indeed, species are adapted to different ecological and environmental ranges. The understanding of biotic diversity begins with an understanding of the tradeoffs that lead to specialisation. These tradeoffs imply that the "jack of all trades is master of none" (Leigh and Wright 1990). An ecological continuum of strategies between those species adapted to very narrow and those adapted to very broad ecological ranges (i.e. specialists vs generalists) exists. Within this continuum, the potentiality of exploiting habitats not only below the critical height $h_{c}$ but also above it, force very different ecological and evolutionary scenarios, in particular, for the optimisation of dispersal or colonisation rates in new available territory, either by increasing resource utilisation scale (O’Neill et al. 1988) or by using different movement strategies (With and King 1999). The main assumptions involved in the dispersal model used to illustrate this fact, are: i) Species habitat definition can integrate both different growth cycle phases (i.e. gap-dynamics stages) as well as different vertical microenvironments. Thus, low-canopy point maps can be related to habitat availability landscapes. ii) Strong habitat dependencies can be established for the survival of some species of the rainforests. iii) Random and local conditions trigger the ultimate dispersal process of the species. And iv) extinction events are negligible once the species are settled in a suitable habitat, or similarly, the rates of colonisation are faster than those of extinction providing an always growing population. These conditions are general enough to cover a wide range of species living in the understory rainforest ecosystem.

Interestingly, the observed $h_{c}$ is near 10 meters, which is the height chosen in field studies to differentiate lowcanopy (light-demanding) from high-canopy (shadetolerant) species (Welden et al. 1991). Low-canopy species able to survive in habitats that can only exist below $h_{c}$ represent specialised species adapted to live in gap-light environments with high sun irradiance. Percolation theory predicts that in random percolating maps with a fraction of available habitat above a gap fraction of $\mathrm{p}_{\mathrm{c}} \approx 0.40725$, species can move freely through the landscape (O'Neill et al. 1988). However, in more aggregated landscapes (i.e. such as those below $h_{c}$ in $\mathrm{BCI}$ ) this value may increase because aggregation diminishes connectivity at long distances, and thus, more species can potentially coexist below $h_{c}$ compared to a random landscape with the same $\mathrm{N}_{\omega}(\mathrm{h})$.

Species with suitable habitats below $h_{c}$ will always experience a strongly correlated and sparse landscape, and therefore may be very sensitive to landscape transformations involving both habitat loss and habitat fragmentation (i.e. loss of aggregation in spatial habitat distribution). Indeed, temporal fluctuations in gap formation and canopy recovery are much higher at lower heights in the forest. In such dynamic landscapes there is a lower survival probability in those species whose dispersal rate or, consequently, resource utilisation scale (O'Neill et al. 1988), are not large enough to overcome the changing structure of the landscape (Keymer et al. 2000, Johst et al. 2002). Therefore, strong evolutive pressures should exist to reinforce those mechanisms allowing the adaptation of dispersal strategies to a sparse and changeable underlying landscape. Thus, their dispersal strategies should be of the Lévy type (i.e. low Lévy indexes) and some plasticity should be necessary to adapt dispersal strategies to landscape changes. Colonisation of new available territory must involve few dispersal events but with large-distance coverage (i.e. long-distance dispersal allows the accessibility to far habitat aggregates). Moreover, directed dispersal strategies (e.g. animal seed dispersal) allows for a better adjustment of new habitat colonisation to a changeable landscape. As an example, dispersal strategies of lightdemanding plant species are commonly directed by animals (Leigh and Wright 1990). Thus, below $h_{c}$, 
Lévy-type strategies based on directed mechanisms of dispersal should have a strong adaptive value. Species with such dispersal strategies should be more sensitive to habitat loss (which is already sparse) than to local habitat fragmentation.

On the other hand, species covering a sufficiently large range of environmental conditions providing some vertical microenvironments or some gap-dynamic stage with maximum canopy heights above $h_{c}$, will experience a fully connected landscape of potential suitable habitats through all the landscape. For example, shade-tolerant plant species (and associated fauna) capable of living in habitats not only below but also above $h_{c}$ will experience a fully connected landscape. Two major consequences may be derived from this ecological situation: i) longdistance dispersal and directed dispersal is not strictly necessary, and ii) once in a connected landscape dispersal strategy doesn't need to be changed. Indeed, optimal dispersal strategies above $h_{c}$ involve: i) a high number of short-scaled dispersal events (i.e. higher Lévy indexes than for species living in sparse landscapes, Fig. 3, left), and ii) independency from the underlying amount of available habitat in the landscape. The latter fact is explained because of a percolation effect. Although at increasing cutoff heights the amount of available habitat increases, the dispersal strategy doesn't change. This is because optimal dispersal strategies mainly depend on the connectivity of habitat patches and not on the quantity of habitat. Once reached the percolation threshold, all the available habitat becomes connected, and this fact determines a unique dispersal strategy (i.e. a unique Lévy index) emerging as optimal for all the low-canopy maps above $\mathrm{h}_{\mathrm{c}}$ (Fig. 3, right).

The evolutive costs of showing a greater physiological or behavioural plasticity (i.e. larger ranges of environmental conditions for survival) could be compensated by the fact that in fully connected landscapes, dispersal strategies should not necessarily be so efficient: shortdistance and non-directed dispersal might be good enough to explore new territories. (e.g. dispersal strategies of shade-tolerant plant species are commonly driven by the wind, Leigh and Wright 1990). Generalist species have more available habitat by definition. Therefore, they should be less sensitive to habitat loss and habitat dynamical changes than highly specialized species. However, generalist species within a fully connected landscape could have evolved short-ranged and nondirected dispersal strategies and as a consequence could be much more sensitive to local fragmentation processes (i.e. loss of aggregation in spatial habitat distribution) when spreading to new territories.

Changes in the gap-dynamics of rainforests may modify the value of $h_{c}$ and thus the relative potential number of species that are more sensitive to habitat loss (below $h_{c}$ ) or to fragmentation processes $\left(\right.$ above $h_{c}$ ), imposing important constrains to biodiversity patterns.
Moreover, the presence of a critical height $\left(h_{c}\right)$ may influence the competitive outcomes of closely related species. Competition between philogenetically related species (i.e. similar ecological ranges) can promote coexistence only when the less competitive one has evolved dispersal strategies capable to overcome its competitive disadvantage. That seems to be the case in the disjoint vertical distribution of lizards in Puerto Rico (Reagan 1992, Leal et al. 1998). In such cases, the presence of a percolating critical height $\left(h_{c}\right)$ should be decisive in the final competitive outcome. Once it is known $h_{c}$, some other practical consequences for rainforest biodiversity conservation could be derived. For example, the control of an invading species in the rainforest canopy should be managed in a very different way if their potential suitable habitats are all below $h_{c}$, or instead some of them are above.

We believe that a more integrative approach to the definition of rainforest species habitats, providing a more global and probabilistic approach to the study of the structure and functioning of rainforests, may be helpful to the understanding of some robust regularities at the whole system level. Such a new approach should allow us to establish new links between different components of the system (i.e. species, functional groups, etc.) or to compare different systems (i.e. different rainforests around the world) by means of objective measures. Moreover, we hope that this kind of approach may give further insights to the understanding of rainforest understory complexity by guiding new field studies, or integrating in a more general framework the already existent huge collection of case studies devoted to concrete rainforest species.

Acknowledgements - The authors thank Rick Condit for providing us with BCI data. We also thank David Alonso and William Parcher for useful discussions. This work has been supported by a grant PB97-0693 (JGPG) and by the Santa Fe Institute (RVS).

\section{References}

Andren, H. 1994. Effects of habitat fragmentation on birds and mammals in landscapes with different proportions of suitable habitat: a review. - Oikos 71: 355-366.

Baker, P. J. and Wilson, J. S. 2000. A quantitative technique for the identification of canopy stratification in tropical and temperate forests. - For. Ecol. Manage. 127: 77-86.

Bascompte, J. and Solé, R. 1996. Habitat fragmentation and extinction thresholds in spatially explicit models. - J. Anim. Ecol. 65: 465-473.

Bartumeus, F., Peters, F., Pueyo, S. et al. 2003. Helical Lévy walks: adjusting searching statistics to resource availability in microzooplancton. - Proc. Natl Acad. Sci. USA 100: $12771-12775$.

Becker, P., Rabenold, P., Idol, J. et al. 1988. Water potential gradients for gaps and slopes in a Panamanian tropical moist forest's dry season. - J. Trop. Ecol. 4: 173-184.

Bunn, A., Urban, D. and Keitt, T. H. 2000. Landscape connectivity: a conservation application of graph theory. - J. Environ. Manage. 59: 265-278. 
Chesson, P. and Pantastico-Caldas, M. 1994. The forest architecture hypothesis for diversity maintenance. - Trends Ecol. Evol. 9: 79-80.

Condit, R. 1998. Tropical forest census plot. - Springer-Verlag.

Denslow, J. 1987. Tropical rainforest gaps and tree species diversity. - Annu. Rev. Ecol. Syst. 18: 431-451.

Drake, J., Dubayah, R., Clark, D. et al. 2002. Estimation of tropical forest structural characteristics using large-footprint lidar. - Remote Sensing Environ. 79: 305-319.

Gamarra, J. G. P. and Solé, R. V. 2002. Biomass-diversity responses and spatial dependencies in disturbed tallgrass prairies. - J. Theor. Biol. 215: 469-480.

Gardner, R., Milne, B. and O'Neill, R. 1987. Neutral models for the analysis of broad-scale landscape patterns. - Landscape Ecol. 1: 19-28.

Gilbert, L. 1980. Food web organization and the conservation of Neotropical diversity. - In: Soule, M. E. and Wiclox, B. A. (eds), Conservation biology. Sinauer Associates, pp. 1133.

Hubble, S. P. and Foster, R. 1986. Canopy gaps and the dynamics of a Neotropical forest. - In: Crawley, M. J. (ed.), Plant ecology. Blackwell Scientific, pp. 77-96.

Iwasa, Y. 2000. Lattice models and pair approximation in ecology. - In: Dieckman, U., Law, R. and Metz, J. A. J. (eds), The geometry of ecological interactions: simplifying spatial complexity. Cambridge Univ. Press, pp. 227-247.

Johst, K., Brandl, R. and Eber, S. 2002. Metapopulation persistence in dynamic landscapes: the role of dispersal distance. - Oikos 98: 263-270.

Katori, M., Kizaki, S., Terui, Y. et al. 1998. Forest dynamics with canopy gap expansion and stochastic Ising model. - Fractals 6: 81-86.

Keitt, T., Urban, D. and Milne, B. 1997. Detecting critical scales in fragmented landscapes. - Conserv. Ecol. [online], 1, 4. URL http://www.consecol.org/voll/iss1/art4.

Keymer, J. E., Marquet, P., Velasco-Hernandez, J. X. et al. 2000. Extinction thresholds and metapopulation persistence in dynamic landscapes. - Am. Nat. 156: 478-494.

Kubo, T., Iwasa, Y. and Furumoto, N. 1996. Forest spatial dynamics with gap expansion: total gap area and gap size distribution. - J. Theor. Biol. 180: 229-246.

Leal, M., Rodriguez-Robles, J. and Losos, J. 1998. An experimental study of interspecific interactions between two Puerto Rican Anolis lizards. - Oecologia 117: 273-278.

Leigh, J. E. 1999. Dramatis personae. - In: Leigh, J. E. (ed.), Tropical forest ecology: a view from Barro Colorado Island. Oxford Univ. Press, pp. 15-45.

Leigh, J. E. and Wright, S. 1990. Barro Colorado Island and tropical biology. - In: Gentry, A. H. (ed.), Four Neotropical rainforests. Yale Univ. Press, pp. 28-47.

Loehle, C., Li, B. and Sundell, R. 1996. Forest spread and phase transitions at forest-prairie ecotones in Kansas, USA. - Landscape Ecol. 11: 225-235.

Marell, A., Ball, J. and Hofgaard, A. 2002. Foraging movement paths of female reindeer: insights from fractal analysis, correlated random walks, and Lévy flights. - Can. J. Zool. 80: $854-865$.

Milne, B., Johnson, A., Keitt, T. et al. 1996. Detection of critical densities associated with pinon-juniper woodland ecotones. - Ecology 77: 805-821.

Molino, J. F. and Sabatier, D. 2001. Tree diversity in tropical rain forests: a validation of the intermediate disturbance hypothesis. - Science 294: 1702-1704.

O’Neill, R., Milne, B., Turner, M. et al. 1988. Resource utilization scale and landscape pattern. - Landscape Ecol. 2: $63-69$.

Parker, G. and Brown, M. 2000. Forest canopy stratification - is it useful? - Am. Nat. 155: 473-484.

Peitgen, H. O., Jürgens, H. O. and Saupe, D. 1992. Chaos and fractals. New frontiers of science. - Springer-Verlag.
Pelt, R. and Franklin, J. 2000. Influence of canopy structure on the understory environment in tall, old-growth, conifer forests. - Can. J. For. Res. 30: 1231-1245.

Ramos-Fernández, G., Morales, J., Miramontes, O. et al. 2004. Lévy walk patterns in the foraging movements of spider monkeys Ateles geoffroyi. - Behav. Ecol. Sociobiol. 55: $223-230$.

Reagan, D. P. 1992. Congeneric species distribution and abundance in a three-dimensional habitat: the rain forest Anoles of Puerto Rico. - Copeia 1992: 392-403.

Reagan, D. P. and Waide, R. B. 1996. The food web of a tropical rainforest. - Univ. of Chicago Press.

Richards, P. 1952. The tropical rain forest. - Cambridge Univ. Press.

Runkle, J. 1984. Development of woody vegetation in treefall gaps in a beech-sugar maple forest. - Holarct. Ecol. 7: 157164.

Satake, A., Iwasa, Y., Hakoyama, H. et al. 2004. Estimating local interaction from spatiotemporal forest data, and Monte Carlo bias correction. - J. Theor. Biol. 226: $225-$ 235.

Schlicht, R. and Iwasa, Y. 2004. Forest gap dynamics and the Ising model. - J. Theor. Biol. 230: 65-75.

Schnitzer, S. and Carson, W. 2000. Have we missed the forest because of the trees? - Trends Ecol. Evol. 15: 376-377.

Shelly, T. 1984. Comparative foraging behavior of Neotropical robber flies Diptera: Asilidae. - Oecologia 62: 188-195.

Smith, A. 1973. Stratification of temperate and tropical forests. - Am. Nat. 107: 671-682.

Solé, R. V. 2000. Order, complexity, disorder. - In: Signs of life: how complexity pervades biology. Basic Books, New York, pp. $29-59$.

Solé, R. V. and Manrubia, S. 1995. Are rainforests selforganized in a critical state? - J. Theor. Biol. 173: 31-40.

Stauffer, D. and Aharony, A. 1985. Introduction to percolation theory. - Taylor and Francis.

Terborgh, J. 1985. The vertical component of plant species diversity in temperate and tropical forests. - Am. Nat. 126: $760-777$.

Terborgh, J. 1992. Sunlight and stratification. - In: Diversity and the tropical rain forest. Scientific Americal Library, Freeman and Co, pp. 105-129.

Turner, M., Gardner, R. and O’Neill, R. 2001. Landscape ecology in theory and practice: pattern and process. - Springer-Verlag.

Viswanathan, G., Buldyrev, S., Havlin, S. et al. 1999. Optimizing the success of random searches. - Nature 401: 911-914.

Welden, C., Hewett, S., Hubbell, S. et al. 1991. Survival, growth, and recruitment of saplings in canopy gaps and forest understory on Barro Colorado Island, Panamá. - Ecology 72: $35-50$.

Whitmore, T. 1988. The influence of tree population dynamics on forest species composition. - In: Davy, A. J., Hutchings, M. J. and Watkinson, A. R. (eds), Population biology of plants. Blackwell, pp. 271-291.

Whitmore, T. 1997. An introduction to tropical rain forests. - Oxford Univ. Press.

Wiens, J., Schooley, R. and Weeks Jr., R. 1997. Patchy landscapes and animal movement: do beetles percolate? - Oikos 78: 257-264.

With, K. 1997. The application of neutral landscape models in conservation biology. - Conserv. Biol. 11: 1069-1080.

With, K. 2002. The landscape ecology of invasive spread. - Conserv. Biol. 6: 1192-1203.

With, K. and King, A. 1999. Extinction thresholds for species in fractal landscapes. - Conserv. Biol. 13: 314-326.

Zagt, R. and Werger, M. 1996. Dynamics of tropical communities. - In: Newbery, D. M., Prins, H. H. T. and Brown, N. (eds), Community structure and the demography of primary species in tropical rainforests. Blackwell Science, pp. 193219. 